\title{
Defined nano-structuring with ultrashort pulses in gelatin biopolymer films for tissue-engineering
}

\author{
Chandra S.R. Nathala ${ }^{a, c}$, Albena Daskalova $^{\mathrm{b}}$, Irina Bliznakova ${ }^{\mathrm{b}}$, Stefan Lueftenegger ${ }^{\mathrm{a}}$, Alexandra \\ Zhelyazkova $^{\mathrm{b}}$, Seres Enikoe ${ }^{\mathrm{a}}$, Thomas Ganz ${ }^{\mathrm{c}}$, Wolfgang Husinsky $\mathbf{y}^{\mathrm{a}, *}$ \\ a IAP, Vienna Technical University, Wiedner Hauptstrasse 8-10, A-1040 Vienna, Austria. \\ ${ }^{b}$ Institute of Electronics, Bulgarian Academy of Sciences, 72, Tsarigradsko Chaussee Blvd., 1784, Sofia, Bulgaria. \\ ${ }^{c}$ Femtolasers Produktions GmbH, Fernkorngasse 10, 1100 Vienna, Austria \\ *Corresponding author e-mail address: husinsky@iap.tuwien.ac.at
}

\begin{abstract}
We compare the morphological modifications in gelatin films, induced with an 800nm femtosecond amplifier, in subject of the number of input pulses and the input pulse duration. Foam is formed in all cases, but only under certain conditions the desirable structures that mimic the native subcellular matrix are formed. The number of input pulses significantly changes the foam structure, allowing a well-defined nano-structuring for optimal tissue-engineering.
\end{abstract}

\section{Introduction}

Tissue Engineering requires the use of a temporary porous matrix in order to guide the regeneration of tissue to the desired shape. Dense cavitation and bubbling phenomenon yield micro/nano-foaming when biopolymer films like collagen and gelatine are irradiated with laser pulses ${ }^{[1,2]}$. It is hypothesized that such artificially created scaffolds matrix may be seeded with cells which then are encouraged to develop into the desired tissue. The dependence of laser fluence, pulse duration, wavelength and sample concentration on structure formation on stand-alone collagen and gelatin films has been already reported ${ }^{[3]}$. In this work we explore the dependence of pulse number on the structure formation.

\section{Experiment}

For preparation of self-standing gelatin films of $\sim 100$ microns thickness, $1.6 \mathrm{~g}$ of gelatine is dissolved in $20 \mathrm{mg}$ of distilled water, which is then heated up to $60^{\circ} \mathrm{C}$. The resulting viscous solution was shuffled for 35 minutes at this temperature to fully dissolve the gelatine. A thin layer of this solution is then applied on microscopic slides. The films thus prepared are irradiated in air by a $1 \mathrm{KHz}, 800 \mathrm{~nm}$ femtosecond laser amplifier (Femtopower) in subject of different fluences, pulse durations, and number of pulses. The pulse duration is varied by changing the prism spacing in the compressor part of the amplifier. The number of pulses (spaced 1ms apart) are extracted by controlling the internal pockel-cell of the amplifier.

\section{Results and Discussions}

Here we present the modifications of the gelatin film, irradiated with a fixed fluence of $3 \mathrm{~J} / \mathrm{cm}^{2}$ in subject to the pulse duration (30fs, $100 \mathrm{fs}$ and 5ps) and number of pulses (1, 2, 5, 25, 50 and 100). Fig. 1 shows the SEM images taken at the irradiated regions with $30 \mathrm{fs}$ (Fig.1a to f) and 100fs (Fig.1g to 1).
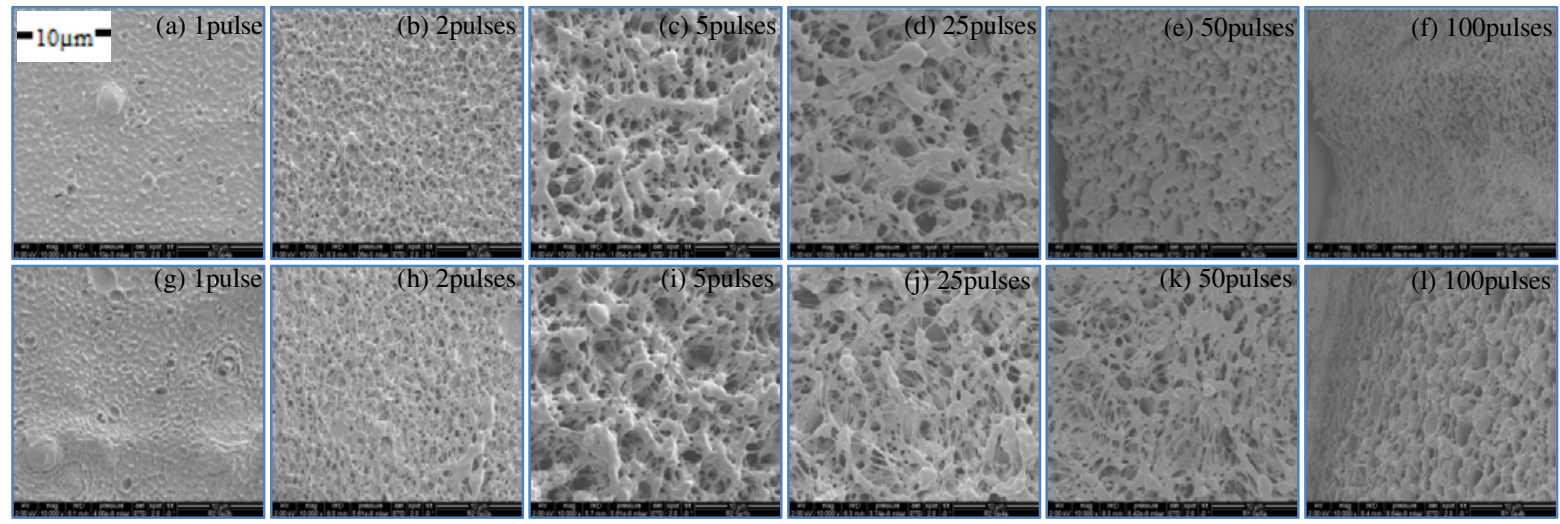

Fig. 1. SEM images of gelatin thin films after irradiation with 30fs pulses (a to f) and 100fs pulses (g to l). Fluence: $3 \mathrm{~J} / \mathrm{cm}^{2}$ 
Fig.1 clearly shows the onset of formation of a network of interconnected micro-pores with the number of irradiated pulses. We can clearly distinguish the onset of surface modification which starts by irradiation with 1 pulse, and after irradiation with more than 25 pulses the surface modification transforms to ablation of the fine structures.

Fig. 1 reveals further, that the pulse duration doesn't have such an influence like the number of input pulses. To investigate the pulse duration dependency in more detail we performed an identical measurement but with a $5 \mathrm{ps}$ pulses, see Fig. 2. No modifications were observed when the sample is irradiated with 1pulse at $5 \mathrm{ps}$ pulse duration at $3 \mathrm{~J} / \mathrm{cm}^{2}$ fluence.

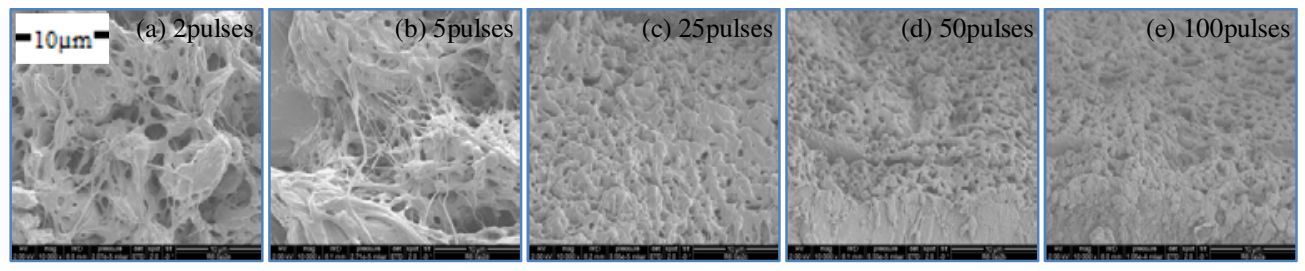

Fig. 2. SEM images of gelatine films after irradiation with a $5 \mathrm{ps}$ pulse. Fluence: $3 \mathrm{~J} / \mathrm{cm}^{2}$

Fig. $3 \mathrm{a} \& \mathrm{~b}$ shows the thickness of the thin inter-connected nano-pores and cavity sizes respectively. It is observed that these sizes depend strongly on the pulse number.

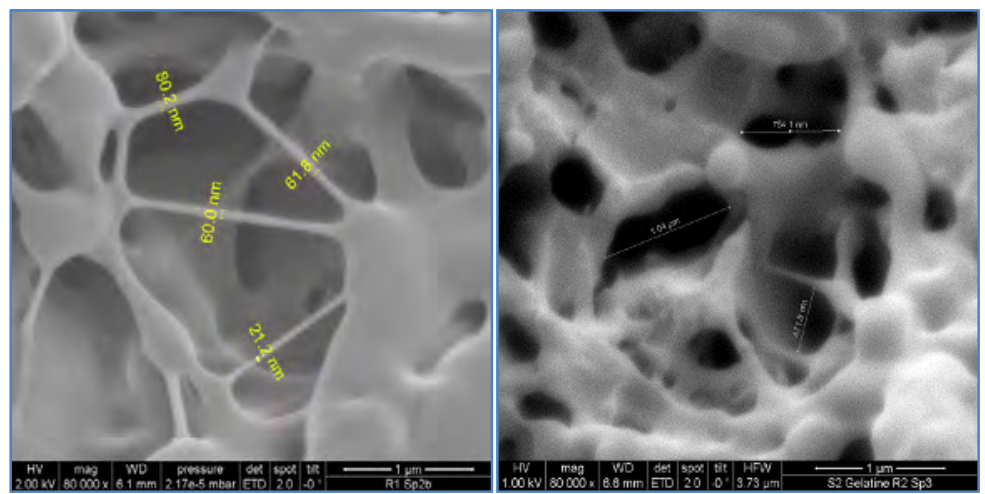

Fig.3. SEM images of gelatine thin film at 80,000 magnification showing dimensions of thin interconnects and cavities.

The choice of femtosecond pulses may be more preferable for controlled and defined nanostructuring in biopolymers. The nano-structuring, respectively the formed cavitations, bubbles and nanofibers, depends significantly on the number of input pulses. This dependency allows a tuning of the bubble and cavitations size and enables a simple way to find the optimal structure for tissue engineering. The measurements indicate that probably a number between $5-25$ input pulses may be most suitable for tissue engineer. In a next step cells will be grown on the modified biopolymers films.

\section{Acknowledgment}

This work was supported by the Österreichische Forschungsfödergesellschaft (FFG) (Project 834325) and the Bulgarian National Science Fund (NSF). (Project DMU 03/35/2011). Authors would like to thank Ms.Karin Whitmore for her patience in imaging the samples.

\section{References}

[1] S. Lazare, V.Tokarev, A.Sionkowska, M.Wisniewski, "Surface foaming of collagen, chitosan and other biopolymer films by KrF excimer laser ablation in the photomechanical regime", Appl.Phys.A, Vol 81., 465-470 (May 2005)

[2] S.Lazare, R.Bonneau, S.Gaspard, M.Oujja, R.De Nalda, M.Castillejo, A.Sionkowska, "Modeling the dynamics of one laser pulse surface nanofoaming of biopolymers", Appl.Phys.A, Vol 94., 719-729., (March 2009)

[3] Marta Castillejo, Rebeca de Naida, Mohamed Oujja, Mikel Sanz, "Wavelength Effects in Femtosecond Pulsed Laser Ablation And Deposition", AIP Conf.Proc.1278, 303 (2010) 